\title{
Analyzing controller conflicts in multimodal smart grids - framework design
}

\author{
Astrid Nieße ${ }^{*}$ and Arash Shahbakhsh \\ From The 7th DACH+ Conference on Energy Informatics \\ Oldenburg, Germany. 11-12 October 2018
}

\footnotetext{
* Correspondence: niesse@ei.unihannover.de

Group Energy Informatics, Leibniz University Hanover, Hannover, Germany
}

\begin{abstract}
In future energy systems dominated by distributed energy resources, additional flexibility potential will be unlocked by interlinking the infrastructures of different energy systems. Direct and indirect coupling of different energy systems like power grids, gas and heat infrastructures leads to multimodal energy systems. Combined with an ongoing pervasion of the resulting interconnected system with information and communication technology (ICT), multimodal smart grids evolve. The pervasion of the system with autonomous controllers leads on to the development of a distributed adaptive system. From an engineering perspective, it shows the desired characteristics regarding scalability and self-stabilization. Unlike these desired aspects, other effects can emerge from the distributed and adaptive nature of this system: Autonomous controllers might counteract, thus leading to instable system states. From the perspective of distributed systems research, these controller conflicts can thus be understood as unintended effects of emergence. In this short paper, we present design decision for a framework that will be developed for the evaluation of controller conflicts in multimodal smart grids. The concept of technical emergence will be applied to deterministically simulate controller conflicts generated by autonomous controllers of interlinked infrastructures.
\end{abstract}

Keywords: Controller conflicts, Multimodal energy systems, Smart grids, Multi-agent systems, Emergence

\section{Introduction}

Four current developments characterize the ongoing and upcoming changes in the current stage of evolution as indicators of a transitional stage to distributed and multimodal smart grids. Thus, they can be understood as transition driving forces:

First, the generation of electricity is shifted from large capacities in the high voltage grid to small units in the distribution grid (Appelrath et al., 2012). Second, the flexibility potential of small loads in the distribution grid can be added to compensate for missing stabilizing capacity in the high voltage grid (Stadler et al., 2007). Third, whereas autonomous controllers have been an important asset in transmission grids for many years (Lehnhoff et al., 2011) more and more autonomous controllers of different types are now installed in distribution grids (Nguyen et al., 2011). Fourth, additional flexibility potential will be unlocked by interlinking the infrastructures of

(c) The Author(s). 2018 Open Access This article is distributed under the terms of the Creative Commons Attribution 4.0 International License (http://creativecommons.org/licenses/by/4.0/), which permits unrestricted use, distribution, and reproduction in any medium, provided you give appropriate credit to the original author(s) and the source, provide a link to the Creative Commons license, and indicate if changes were made. 
different energy systems. Originally unconnected energy systems are dynamically coupled (Lehnhoff et al., 2013).

Combined with an ongoing pervasion of the resulting interconnected system with information and communication technology (ICT), multimodal smart grids evolve to guarantee flexibility and control of a complex system under severe reliability and resilience requirements. The first, second and third transition driving forces enlarge the degree of distribution of the energy system regarding electricity generation, usage and grid operation. This transition follows different steps, including (1) the distribution of electricity generation, (2) the usage of distributed energy units for system stability needs, and (3) an autonomous operation of the system through cooperating autonomous units, with central systems to ensure reliability and control in cases of emergency (Appelrath et al., 2012; Negenborn et al., 2010). The pervasion of the energy system with autonomous controllers leads on to the development of a distributed adaptive system. From an engineering perspective, it shows the desired characteristics regarding scalability and self-stabilization. Unlike these desired aspects, other effects can emerge from the distributed and adaptive nature of this system: Autonomous controllers might counteract, thus leading to unintended system behavior like oscillations and instable system states within a multi-controller system (van Breemen, 2001). From the perspective of distributed systems research, these controller conflicts can thus be understood as unintended effects of emergence (GDM et al., 2005).

With the fourth transition driving force - the evolvement of multimodal smart grids - these unwanted effects might extend and multiply: Whereas within one sector like electricity grids, the different controllers might in theory be compatible during the design process, the interlinkage of different energy infrastructures has not been part of the design process. As the reliability and resilience of energy systems is of crucial importance for industrial societies, approaches are needed to increase the confidence in the upcoming largely adaptive distributed system.

Up to now, research in this field has been focused on electrical networks (Martins, 1999), using either analytical modeling or simulation case studies based on detailed models. In the first case, the investigation of controller conflicts based broadly on a comprehensive mathematical modeling of the physical components and controllers (Roy \& Pota, n.d.; Bindeshwars \& Sharma, n.d.; Meliopoulos et al., 2006). These methods cannot be applied to complex systems like multimodal smart grids. In the latter case, the reported controller conflicts have been identified in simulation studies. In (Velasquez et al., 2017) an electrical power system, its components and associated controllers are modeled in different software, programs and platforms. The model is finally investigated with various scenarios, strictly spoken, this aspect identifies controller conflicts by chance or from previous experience.

The significant features of our evaluation approach in comparison to the aforementioned studies are as follows: First, to the best of our knowledge, there is no report about the investigation of controller conflicts in multimodal energy systems. Second, this study embodies self-organized (adaptive) and smart controllers. Third, some techniques will be developed in this project which systematically generate controller conflicts. Fourth, new performance metrics for the multimodal smart grid will be formulated which are preliminary for a systematic identification of controller conflicts. 
The core of the research within this project is to study controller conflicts in multimodal smart grids using the concept of emergence in technical systems as simulation paradigm. As a first step, a simulation framework has to be designed that is appropriate to support this methodological approach. In this contribution, we present design considerations on this framework.

The rest of this contribution is structured as follows: In the first section the research focus is described in detail. In the second section, we present the framework architecture. Based on the research goals and the introduced framework, the procedure of the evaluation studies on controller conflicts is outlined in the layered system in the next three sections. Conclusions and planned work is outlined in the last section.

\section{Research focus}

The main objective of the proposed project is the analysis of controller conflicts manifesting in multimodal smart grids dominated by various autonomous controllers. For this objective, an excerpt of the combined energy system - composed of a power distribution grid, distributed energy resources, flexible loads, and heating systems with the exemplary relevant coupling points - combined heat and power plants, gas distribution grids, and gas pumping stations - will be modeled including the autonomous controllers as expected in the field for the year 2050 (Niedersächisches Ministerium für Umwelt, Energie und Klimaschutz, n.d.). The research aspiration of the project is to answer the following three research questions (RQ).

RQ 1: How can controller conflict situations in multimodal smart grids be identified and distinguished from other system states with insufficient performance?

RQ 2: What kind of controller conflicts can manifest during runtime in multimodal smart grids and in which system states do they occur?

RQ 3: Can self-organized multi-agent systems present a suitable approach for modeling controller conflicts?

External events such as a short circuit in power line or change in wind speed force autonomous power system controllers to react, tune up against the events and move the system back to a stable state. These controllers will be modeled as self-organizing agents, thus reflecting the autonomous characteristics of the controllers they represent. They can modify their behavior during runtime for a more efficient performance as a wanted effect. Mutually and from undesired effects of emergence, the interaction of agents could cause an undesirable system behavior in form some unstable states.

\section{Framework design}

In this section, the methodology of work will be introduced. Using the technological basis of a multi agent system, an exemplary system will be modeled as adaptive multi-controller system combined with a smart energy system simulation framework. With the modeling approach of a self-organized multi-agent system, the system offers an abstraction layer regarding single controller types. This allows for an efficient automation of the scenario adaptation needed for an intense simulative scenario exploration, thus identifying the needed set of unstable system states. 
The proposed project follows an artefact-oriented research method to ensure replicability and stepwise evaluation possibilities as has been proposed in (Nieße et al., 2014) and established research recommendations (Hevner \& Ram, n.d.). For this reason, each research question is either connected to an artefact implemented to setup the simulation and software system needed to answer the research question, or results from analyses yielded from using this simulation and software system. These artefacts are shown in Fig. 1. In the following, the three layers of the planned research and simulation framework will be outlined briefly.

\section{Layer of physical component simulation}

As a basis for all later investigations, the physical system has to be defined and modeled. The general goal of this modeling is to set up the multimodal smart grid simulation as the object of observation. Implementing the idea of the smart grid architectural model (SGAM) 2nd IEEE PES International Conference and Exhibition on Innovative Smart Grid Technologies (ISGT Europe), 2011: 5-7 Dec. 2011, Manchester, United Kingdom (2011), the resulting simulation system can be understood as an instance of the component layer. In addition to the electrical system, different heating systems and gas networks have to be modeled as well as heat sinks and

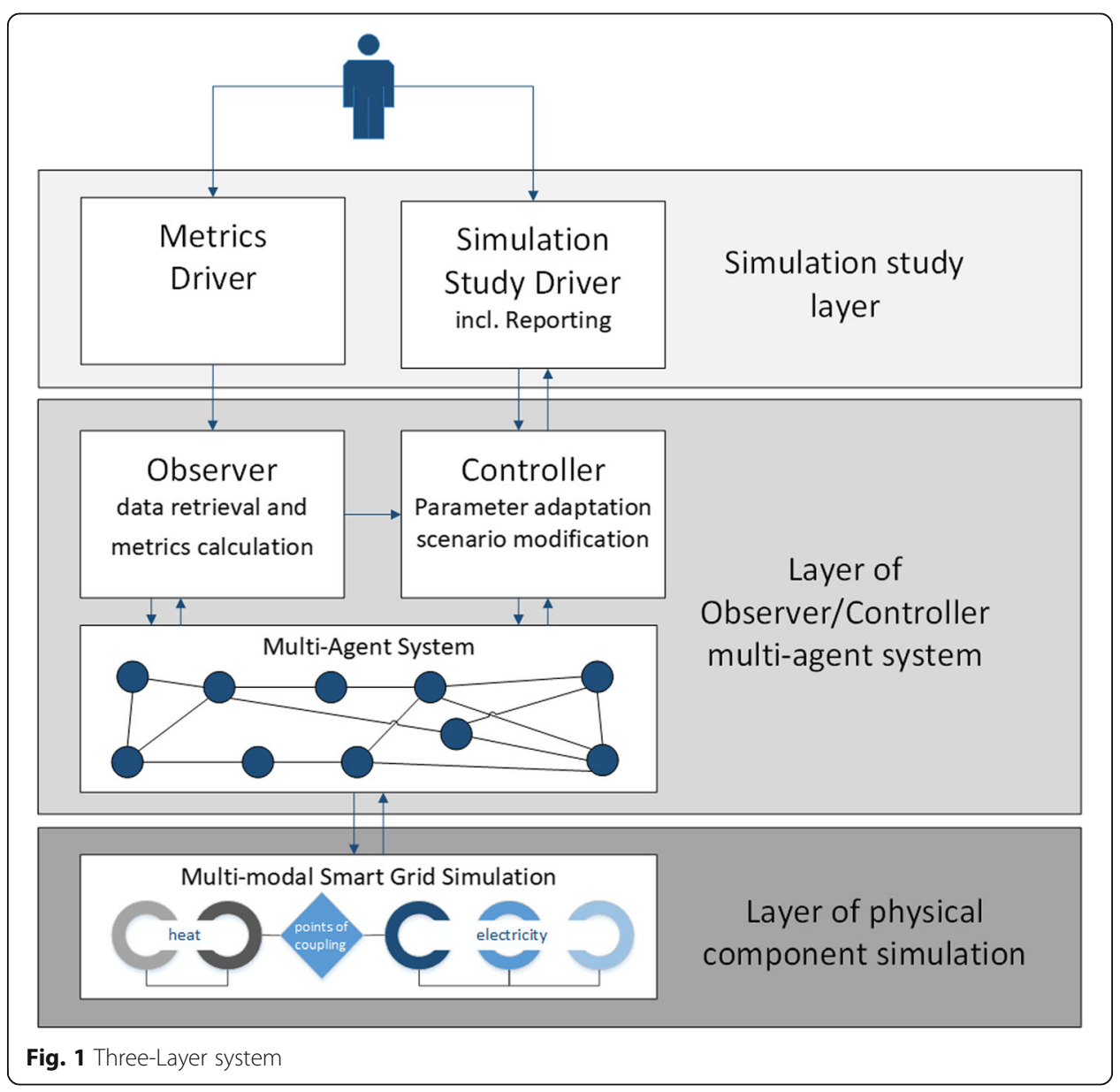


coupling points. Co-simulation tools will be used to integrate various simulation models from different domains.

\section{Layer of observer/controller multi-agent system}

With the Observer/Controller multi-agent system (see second lower layer in Fig. 1) an agent abstraction layer is added to the layer of physical component simulation to represent autonomous controllers and other energy system components as defined in layer 1. The resulting agent abstraction layer is needed for several purposes: First, this layer allows abstraction from the precise and specific view on the modeled energy systems. The abstraction method using agents thus allows to integrate the system model in the intense and automated simulation studies needed to identify unstable system states that result from controller conflicts (Ringler et al., 2016). Second, the system represents an agent-based control system as depicted in Fig. 1. With this purpose in mind, later mitigation actions for controller conflicts can be integrated in the system without changes in the overall design.

With the Observer/Controller approach, a generic architecture has been proposed for the development of self-organizing systems based on multi-agent systems. The main purpose of this architecture is to embed a self-organizing system in a control environment and thus allow for both keeping track of the actual performance of the System under Observation and Control and passing triggers to the system components (the agents) to control this performance. Different observation models can be activated during runtime if needed for the task to be solved by the System under Observation and Control. The general approach has been evaluated in different use cases (McCluskey et al., 2016; Müller-Schloer et al., 2011).

\section{Simulation study layer}

The simulation study layer (see the third layer in Fig. 1) is proposed for generating controller conflict scenarios and developing a metrics. In this layer two blocks work parallel and develop each other. The simulation study driver has two main functionalities, scenario generation and scenario modification. Unstable system states in multimodal energy systems are dynamic by definition. To analyze these states though, an approach is needed to deterministically reproduce these system states. The problem is twofold: First, a scenario exploration strategy has to be applied to identify severe (i.e. performance degraded) system states. Second, based on unstable states recognized in the first step, further examinations are necessary to develop metrics that can later be applied to help identify unstable states (RQ3).

\section{Metrics driver}

It has been already stated that a bad system performance does not necessarily hint to a controller conflict - the system might show bad performance independent of the current controller setup. Whereas in the first case an adaptation of the controller parameters might allow to avoid unstable systems states or lead the system to a stable operation again, the second one cannot be influenced. Parallel to the simulation study layer, the goal is to develop metrics as foundation for the identification of those system states that are classified as unstable caused by controller conflicts. One of the adjacent 
questions is if instabilities can be derived as global rules or depend on the current and preceding system states.

In (McCluskey et al., 2016), two different approaches are identified to distinguish a bad system performance resulting from undesired behavior and bad system performance that does not result from a "performance hole". If the system layout allows separating the control system from the simulation system, different control concepts can be applied to a scenario that lead to an unstable system state. If this leads to a better performance, we can assume an undesired system adaptation.

\section{Analysis of controller conflicts}

With the presented concept, the multi-layer experimental framework will be set up and examination objects - i.e. deterministically generated scenarios prone to unstable system states - will have been identified. Metrics will have been developed to differentiate unstable system states that are a result of controller conflicts (undesired adaptation) from general bad system performance.

One method to analyze controller conflicts is to model the development of the system state from the simulated scenario over time. At some point in time, the system state proceeds from stable to unstable, so this transition can be regarded as a phase transition. With the generated metrics developed in the last section, a distinction function is given to discriminate if the set of system states belongs to the set of controller conflict system states or not. For this kind of systems, classifiers based on support vector decoders have been successfully applied (Tax \& Robert, 1999). With the given complexity of interconnected energy systems though, choosing the appropriate set of system attributes to train the support vector is a challenging task. As has been shown for distributed energy scheduling, the quality of the qualifier substantially depends on choosing the appropriate training data (Bremer \& Sonnenschein, 2013). Therefore, a structured approach has to be developed to evaluate different excerpts of simulation attributes for support vector training. The set of attributes will have to consist of both - attributes characterizing the scenario and attributes characterizing the current simulation state, i.e. the system state over time (Blank et al., 2011).

\section{Conclusion and outlook}

In the work at hand, we presented design decisions for a framework that will be developed to analyze controller conflicts in multimodal smart grids using the concept of emergence in self-organizing systems. This framework will be used in a study on controller conflicts that arise in connected energy systems, specifically. This study includes finding and classifying the system states and controller configurations which cause controller conflicts. From a methodological point of view, the question will be answered if multi-agent systems and the concept of emergence in technical systems constitute an appropriate modeling concept for the analysis of controller conflicts. 


\section{About this supplement}

This article has been published as part of Energy Informatics Volume 1 Supplement 1, 2018: Proceedings of the 7th $\mathrm{DACH}+$ Conference on Energy Informatics. The full contents of the supplement are available online at https:// energyinformatics.springeropen.com/articles/supplements/volume-1-supplement-1.

\section{Authors' contributions}

Both authors have read and approved the final manuscript.

\section{Competing interests}

The authors declare that they have no competing interests.

\section{Publisher's Note}

Springer Nature remains neutral with regard to jurisdictional claims in published maps and institutional affiliations.

Published: 10 October 2018

\section{References}

2nd IEEE PES International Conference and Exhibition on Innovative Smart Grid Technologies (ISGT Europe), 2011: 5-7 Dec. 2011, Manchester, United Kingdom. Piscataway: IEEE; 2011

Appelrath H-J, Kagermann H, Mayer C (2012) Future Energy Grid: Migrationspfade ins Internet der Energie. Springer, Berlin, Heidelberg Bindeshwars N.K., Sharma A. N. Tiwari. coordinated control and interaction between FACTS controller (n.d.)

Blank M, Gerwinn S, Krause O, Lehnhoff S (2011) Support vector machines for an efficient representation of voltage band constraints, pp 1-8

Bremer J, Sonnenschein M (2013) Sampling the search space of energy resources for self-organized, Agent-based Planning of Active Power Provision, pp 214-222

GDM S, Gleizes MP, Karageorgos A (2005) Self-organization in multi-agent systems. Knowl Eng Rev 20:165-189. https://doi. org/10.1017/S0269888905000494

Hevner AR, Ram S. Design Science in Information Systems Research (n.d.)

Lehnhoff S, Hager U, Zimmermann T, Rehtanz C (2011) Autonomous distributed coordination of fast power flow controllers in transmission networks, pp 1-8. https://doi.org/10.1109/ISGTEurope.2011.6162647

Lehnhoff S, Rohjans S, Holzer R, Niedermeier F, Meer Hd. Towards a Mapping of Self-Organization Properties and Non-Functional Requirements in the Operation of Future Smart Grids 2013

Martins NC (1999) Impact of the Interactions Among Power System Controls, pp 1-16

McCluskey TL, Kotsialos A, Müller JP, Klügl F, Rana O, Schumann R (eds) (2016) Autonomic road transport support systems. Birkhäuser, Basel

Meliopoulos AP, Sakis, Cokkinides, George J (2006) IEEE Power Engineering Society general meeting, 2006: 18-22 June 2006 , [Montreal, QC, Canada]. IEEE Operations Center, Piscataway

Müller-Schloer C, Schmeck H, Ungerer T (eds) (2011) Organic computing - a paradigm shift for complex systems. Springer Basel AG, Basel

Negenborn RR, Hellendoorn H, Lukszo Z (2010) Intelligent infrastructures. Springer, Dordrecht

Nguyen PH, Kling WL, Ribeiro PF (2011) Agent-based power routing in Active Distribution Networks, pp 1-6. https://doi.org/ 10.1109/ISGTEurope.2011.6162682

Niedersächisches Ministerium für Umwelt, Energie und Klimaschutz. Szenarien zur Energieversorgung in Niedersachsen im Jahr 2050. (n.d.) 1000

Nieße A, Tröschel M, Sonnenschein M (2014) Designing dependable and sustainable Smart Grids e How to apply Algorithm Engineering to distributed control in power systems, pp 37-51

Ringler P, Keles D, Fichtner W (2016) Agent-based modelling and simulation of smart electricity grids and markets - a literature review. Renew Sust Energ Rev 57:205-215. https://doi.org/10.1016/j.rser.2015.12.169

N.K. Roy, H.R. Pota. Investigating the Controller Interactions of Distribution Systems with Distributed Generation (n.d.)

Stadler M, Krause W, Sonnenschein M, Vogel U. The adaptive fridge - comparing different control schemes for enhancing load shifting of electricity demand. 2007

Tax DMJ, Robert PW (1999) Duin Data Domain Description using Support Vectors, pp 251-256

van Breemen AJN (2001) Agent-based multi-controller systems: a design framework for complex control problems. Twente University Press; op, Enschede

Velasquez J, Piech K, Lehnhoff S, Fischer L, Garske S (2017) Incremental development of a co-simulation setup for testing a generation unit controller for reactive power provision. Comput Sci Res Dev 32:3-12. https://doi.org/10.1007/s00450-0160319-2 